Trinity University

Digital Commons @ Trinity

Physics and Astronomy Faculty Research

Physics and Astronomy Department

3-12-2014

\title{
Experimental Tests of Free-Volume Tracer Diffusion in Water and Other Solvents
}

Daniel Spiegel

Trinity University, dspiegel@trinity.edu

Paulses C. Kollie

Trinity University

Scott J. Van Tilburg

Trinity University

Follow this and additional works at: https://digitalcommons.trinity.edu/physics_faculty

Part of the Physics Commons

\section{Repository Citation}

Spiegel, D.R., Kollie, P.C., \& Van Tilburg, S.J. (2014). Experimental tests of free-volume tracer diffusion in water and other solvents. Journal of Chemical Physics, 140(10), 104507-1-104507-4. doi: 10.1063/ 1.4867911

This Article is brought to you for free and open access by the Physics and Astronomy Department at Digital Commons @ Trinity. It has been accepted for inclusion in Physics and Astronomy Faculty Research by an authorized administrator of Digital Commons @ Trinity. For more information, please contact jcostanz@trinity.edu. 


\title{
Experimental tests of free-volume tracer diffusion in water and other solvents
}

\author{
Daniel R. Spiegel, ${ }^{\text {a) }}$ Paulses C. Kollie, and Scott J. Van Tilburg \\ Department of Physics and Astronomy, Trinity University, San Antonio, Texas 78212-7200, USA
}

(Received 2 January 2014; accepted 25 February 2014; published online 12 March 2014)

\begin{abstract}
Using forced Rayleigh scattering, the tracer diffusion of methyl red through water and eight other solvents at different temperatures is investigated and the results are compared to the Cohen-Turnbull theory of free-volume diffusion. In seven solvents the effective non-Arrhenius activation energy measured experimentally agrees with the Cohen-Turnbull energy. In water, however, the diffusion can be described mathematically by the free volume model but there is a disagreement of more than an order of magnitude between these energies. We propose that the unique "zero point" free volume forced onto water by the strong hydrogen bonding requires a different mechanism for tracer motion. (C) 2014 AIP Publishing LLC. [http://dx.doi.org/10.1063/1.4867911]
\end{abstract}

\section{INTRODUCTION}

In textbooks ${ }^{1,2}$ the temperature $(T)$ dependence of the molecular tracer diffusion coefficient $D$ in liquids is often treated as a pure-Arrhenius Boltzmann process, where in order to move the tracer molecule must overcome the attraction to its neighbors. If the activation energy $U_{\mathrm{A}}$ is defined in the usual way,

$$
U_{\mathrm{A}}=-\partial \ln (D) / \partial(1 / k T),
$$

where $k$ is Boltzmann's constant, then a pure Arrhenius process is one in which the activation energy is constant:

$$
D=D_{0} \exp \left(-U_{\mathrm{A}} / k T\right) .
$$

The textbook approach has been criticized ${ }^{3}$ as not being consistent with some fundamental principles of chemical kinetics. Cohen and Turnbull ${ }^{4-7}$ proposed a very different approach, where the criterion for tracer motion is instead that a free-volume fluctuation creates a void adjacent to the tracer molecule, which the tracer then moves into. The minimum void volume required for tracer motion is $v^{*}$, expected to be about equal to the van der Waals volume of the tracer. If $v_{\mathrm{f}}$ is the average free volume per solvent molecule at $T$, the CohenTurnbull free-volume diffusion coefficient is given by

$$
D=A \sqrt{T} \exp \left(-\gamma v^{*} / v_{\mathrm{f}}\right) .
$$

The coefficient $\gamma$ accounts for free-volume overlap and should be between $1 / 2$ and 1 . The prefactor is proportional to the gas-phase diffusion coefficient and hence the kinetic speed. ${ }^{8}$ Different values of the constant $A$ have been proposed in the literature. ${ }^{9-13}$

To calculate the average free volume $v_{\mathrm{f}}$, Cohen and Turnbull assume the free volume is zero at the glass transition temperature $T_{0}$. The free volume is then calculated using the thermal expansion of the solvent from $T_{0}$ up to the temperature $T$ of the experiment. If $v_{\mathrm{m}}$ is the mean of the specific volume over that temperature range and $\alpha$ is the coefficient of thermal

\footnotetext{
a)dspiegel@trinity.edu
}

expansion, then the average free volume is given to a very good approximation by

$$
v_{\mathrm{f}}=v_{\mathrm{m}} \alpha\left(T-T_{0}\right),
$$

so that the Cohen-Turnbull diffusion coefficient becomes

$$
D=A \sqrt{T} \exp \left(\frac{-\gamma v^{*}}{v_{\mathrm{m}} \alpha\left(T-T_{0}\right)}\right) .
$$

If one defines a Cohen-Turnbull energy as $U_{\alpha}=\frac{k \gamma v^{*}}{v_{\mathrm{m}} \alpha}, D$ can be written as

$$
D=A \sqrt{T} \exp \left(-\frac{U_{\alpha}}{k\left(T-T_{0}\right)}\right) .
$$

In this work, we wish to investigate the temperature dependence of the tracer diffusion of a solute in a number of different solvents, listed in Table I. We will see if Eq. (6) describes the data, and we will test CohenTurnbull theory by comparing the measured $U$, obtained via $D=A \sqrt{T} \exp \left(-\frac{U}{k\left(T-T_{0}\right)}\right)$, to the Cohen-Turnbull prediction $U_{\alpha}=\frac{k \gamma v^{*}}{v_{\mathrm{m}} \alpha}$. We will be particularly interested in how well tracer diffusion in water can be described by Cohen-Turnbull theory. Temperatures, varied using heated or chilled water, were measured with a thermistor, and as such are believed accurate to better than $0.1 \%$. In all solvents studied except water, the glass transition temperature $T_{0}$ was too small to make a difference in the data analysis, and we never observed the downward deviation from linearity at lower temperatures of $\ln (D \sqrt{T}) v s .1 / k T$ demanded by $T_{0}>0$. In water, we will argue below that the free volume is not zero at the glass transition; in addition, we were not able to fit the water data to Eq. (6) with $T_{0}>0$. We therefore set $T_{0}=0$ for all solvents.

\section{RESULTS AND DISCUSSION}

The tracer molecule we employed is ortho methyl red (MR), shown in Fig. 1. The diffusion of MR was measured experimentally using the technique of forced Rayleigh scattering. ${ }^{14-22}$ Two coherent pulsed "pump" laser beams interfere within the sample to produce a grating of 
TABLE I. Calculation of the Cohen-Turnbull energy $U_{\alpha}=\frac{k \gamma v^{*}}{v_{\mathrm{m}} \alpha}$ for the solvents, using $\gamma v^{*}=0.116 \mathrm{~nm}^{3}$ obtained from MR diffusion in toluene. DMFA, DMSO, and THF stand for dimethylformamide, dimethylsulfoxide, and tetrahydrofuran, respectively.

\begin{tabular}{lcccccccc}
\hline \hline & 1. Acetone & 2. Aceto-nitrile & 3. CCl & 4. DMFA & 5. DMSO & 6. Ethanol & 7. THF & 8. Water \\
\hline$v_{\mathrm{m}}\left(\mathrm{nm}^{3}\right)$ & 0.098 & 0.048 & 0.092 & 0.102 & 0.071 & 0.057 & 0.077 \\
$\alpha\left(\mathrm{K}^{-1}\right)$ & $1.4 \times 10^{-3}$ & $1.4 \times 10^{-3}$ & $1.3 \times 10^{-3}$ & $1.0 \times 10^{-3}$ & $1.0 \times 10^{-3}$ & $1.1 \times 10^{-3}$ & $1.1 \times 10^{-3}$ & $2.1 \times 10^{-4}$ \\
$U_{\alpha}(\mathrm{eV})$ & 0.0713 & 0.149 & 0.086 & 0.095 & 0.142 & 0.161 & 0.118 & 2.82 \\
\hline \hline
\end{tabular}

wavevector $q$. The pump beams are absorbed by the MR and create excited cis states within the bright fringes. The cis and the ground trans states have different refractive indices so that "complementary" phase gratings are produced, in which the trans and cis enhancement regions are $180^{\circ}$ out of phase. As expected for an azobenzene, the cis excited-state lifetime is very long, so that the gratings are washed out via MR diffusion after the pump pulses are removed. The grating decay is monitored using a "probe" laser beam of a different wavelength diffracted from the sample. If the trans and cis grating amplitudes are $A_{\mathrm{t}}$ and $A_{\mathrm{c}}$, the homodyne-detected diffracted intensity falls off with time as ${ }^{17-19}$

$$
\frac{I}{I(0)}=\left[A_{t} \exp \left(-q^{2} D_{\mathrm{t}} t\right)-A_{\mathrm{c}} \exp \left(-q^{2} D_{\mathrm{c}} t\right)\right]^{2},
$$

where $D_{\mathrm{t}}$ and $D_{\mathrm{c}}$ are the trans and cis diffusion coefficients. A typical MR decay profile is shown in Fig. 2. The geometric mean diffusion coefficient $D=\sqrt{D_{t} D_{c}}$ can be obtained from a combination of the first two cumulant rates. ${ }^{18}$ It has been pointed out that the difference between $D_{\mathrm{t}}$ and $D_{\mathrm{c}}$ is largest for hydrogen-bonding solvents, where it does not exceed 20\%. ${ }^{20,21}$ Our apparatus is similar to that of Köhler ${ }^{22}$ and has been described previously, ${ }^{17,18}$ with $488 \mathrm{~nm}$ and $633 \mathrm{~nm}$ pump and probe wavelengths, respectively. The temperature increase due to the absorbed pump beams is on the order of $5 \mathrm{mK}$. MR was dissolved in the solvents at $7 \times 10^{-4} \mathrm{M}$, which is dilute in the sense that MR molecules are separated on average by $13 \mathrm{~nm}$, roughly ten MR diameters. We confirmed that the grating decay was indeed due to diffusion by measuring the diffraction decay rate at different grating spacings and we saw the decay rate was proportional to $q^{2}$.

We first wish to understand to what extent, if any, the tracer diffusion can be described as a pure Arrhenius process. Figure 3 shows the diffusion of MR in three different solvents. Error bars are plus/minus one standard deviation over 3-5 repeated trials. Our temperature change is not dramatic - only about $40 \%$. At first glance, the data therefore appear to be purely Arrhenius. Using just one of the individual plots of Fig. 3, we would be hard pressed to argue the data are not pure Arrhenius when error bars are considered. In all solvents in-

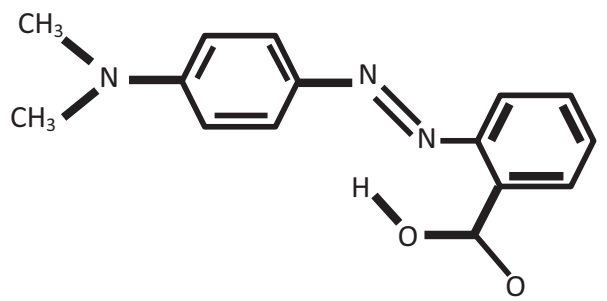

FIG. 1. Ortho methyl red: 2-[4-(dimethylamino)phenylazo]benzoic acid. vestigated, however, we see a small but reproducible upward deviation from the straight line at the lowest temperatures, as shown in Fig. 3. The deviation from linearity can be accounted for with an additional $\sqrt{T}$ term via Eq. (6), as seen in Fig. 3(c). Because the $\sqrt{T}$ term changes by so much less than the exponential for our limited temperature range, the data do not display large deviations from a straight line. From a theoretical perspective, however, pure Arrhenius physics does not apply. We will therefore always find $U$ from the constant slope of $\ln (D / \sqrt{T}) v s .1 / k T$, in keeping with Eq. (6). We find that the slope of $\ln (D / \sqrt{T})$ is about $12 \%$ less than the Arrhenius $\ln (D)$ slope.

In Table I we show the calculation of the Cohen-Turnbull energy $U_{\alpha}$ for each of the solvents employed. $v_{\mathrm{m}}$ is the mean of the van der Waals volume and the specific volume $\frac{M}{\rho N_{A}}$ at $20^{\circ} \mathrm{C}$, where $M$ and $\rho$ are the molecular weight and density of the solvent and $N_{\mathrm{A}}$ is Avogadro's number. The thermal expansion coefficient for water is the mean of $\alpha$ over the temperature region $277 \mathrm{~K}<T<353 \mathrm{~K}$, bounded by the maximum temperature in our water studies, for which $\alpha$ is positive. ${ }^{23}$ Macedo and Litovitz ${ }^{24}$ have argued that diffusion in hydrogen-bonding solvents could be described by a hybrid process in which both Boltzmann bond-breaking and CohenTurnbull free-volume diffusion are important. We therefore calculate $\gamma v^{*}=0.116 \mathrm{~nm}^{3}$ for MR using a measured $U$ $=0.100 \mathrm{eV}$ in toluene, which allows almost no hydrogen bonding. This $\gamma v^{*}$ appears reasonable in view of Fig. 1.

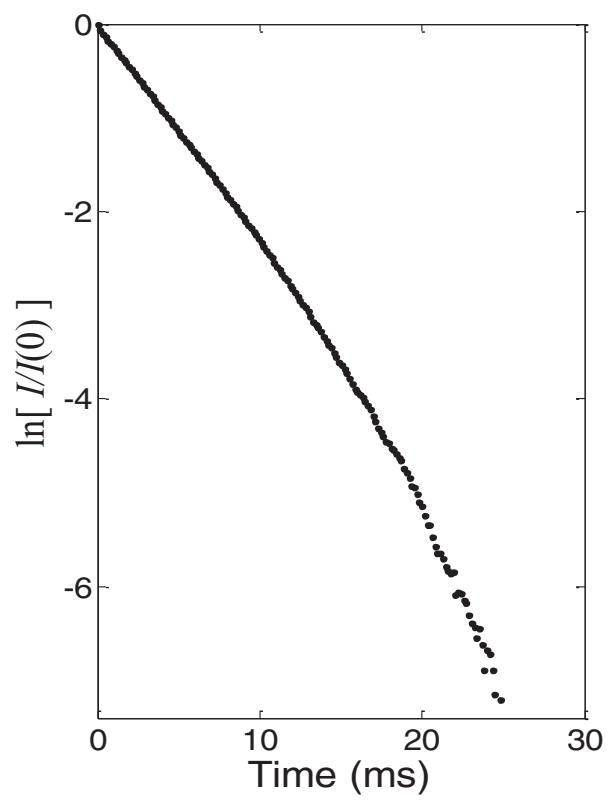

FIG. 2. Forced Rayleigh scattering signal for MR diffusing through $\mathrm{CCl}_{4}$ at $20^{\circ} \mathrm{C}$. 

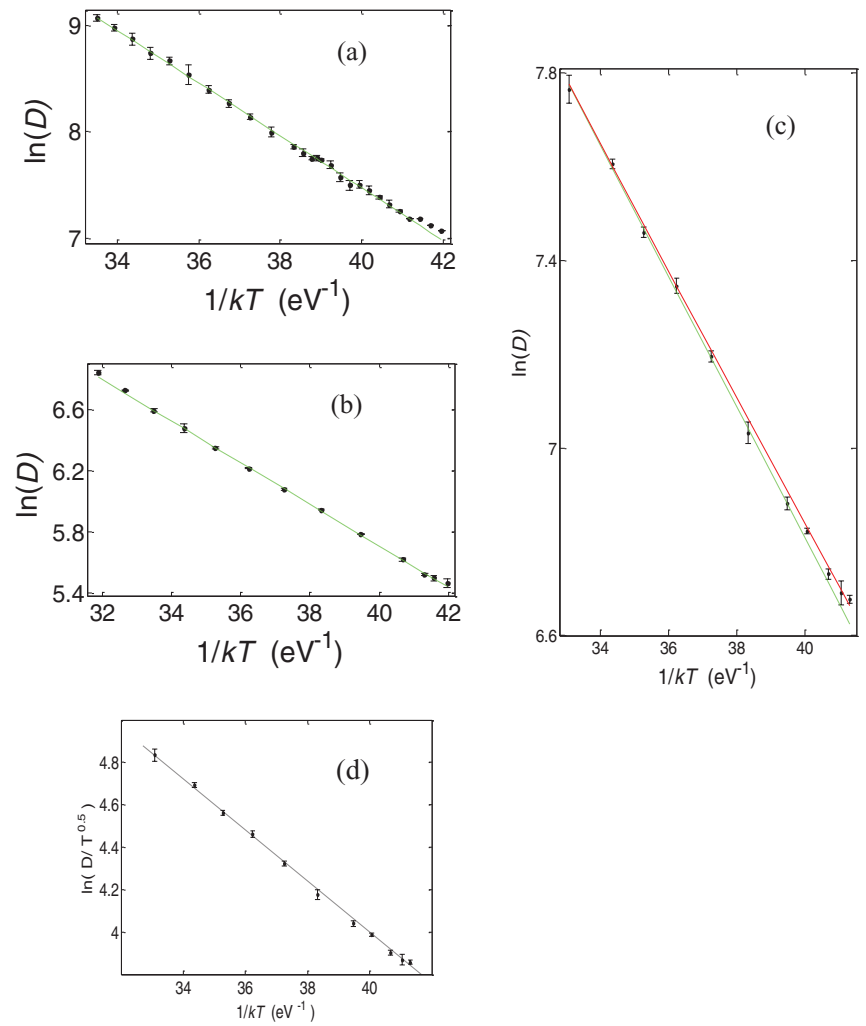

FIG. 3. Tracer MR diffusion of $D\left(\mu \mathrm{m}^{2} / \mathrm{s}\right)$ in (a) ethanol, (b) DMFA, and (c) acetonitrile. The dashed green line is a linear fit of $\ln (D)$ to the hightemperature data and the solid red curve is a fit to the Cohen-Turnbull Eq. (6) with $T_{0}=0$. Fig. 3(d) shows the linear fit to the Cohen-Turnbull equation in acetonitrile more explicitly. The slope of the black dashed line in (d) is $-U$.

Employing this, the values of the Cohen-Turnbull $U_{\alpha}$ for the other solvents are listed in the table.

We now wish to compare the measured values of $U$ to the Cohen-Turnbull $U_{\alpha}$ in Table I. Figure 4 displays the ratio $U / U_{\alpha}$ for each of the solvents. In water it was necessary to use $\mathrm{pH} 10$ because protons attach to the azo group of MR and destabilize the excited state. ${ }^{21}$ (MR is a well-known $\mathrm{pH}$ indicator.) For all solvents except water, $U$ differs from $U_{\alpha}$ by $40 \%$ or less. This difference can be accounted for rather simply if the free-volume overlap factor $\gamma$ is different for the different solvents. The ordinate of Fig. 4 is then the ratio of $\gamma$ for the solvent to that of toluene. For water, however, $U$ is more than an order of magnitude smaller than $U_{\alpha}$. Water has a much smaller molecular size and thermal expansion coefficient than the other solvents, and yet has an experimental $U$ which is only $7 \%$ different from that of ethanol. The $U / U_{\alpha}$ of water cannot be accounted for by $\gamma$, which is between $1 / 2$ and 1 , nor by the hybrid model of Macedo and Litovitz, which demands $U>U_{\alpha}$. Anomalous transport and thermodynamic properties of water have been studied for decades. ${ }^{23,25-28}$ Many of the anomalies are due to strong hydrogen bonding, ${ }^{27,28}$ and we believe that is the case presently. The importance of hydrogen bonding in controlling the self-diffusion of water has been emphasized by Gillen et al. ${ }^{29}$ Angell et al.,${ }^{30}$ Prielmeier et $a l,{ }^{31}$ and Debenedetti and Stanley, ${ }^{28}$ while Lokotosh et al. ${ }^{32}$ have argued for the importance of "crystal-like" hydrogenbonded clusters. The specific volume of water has a clas-

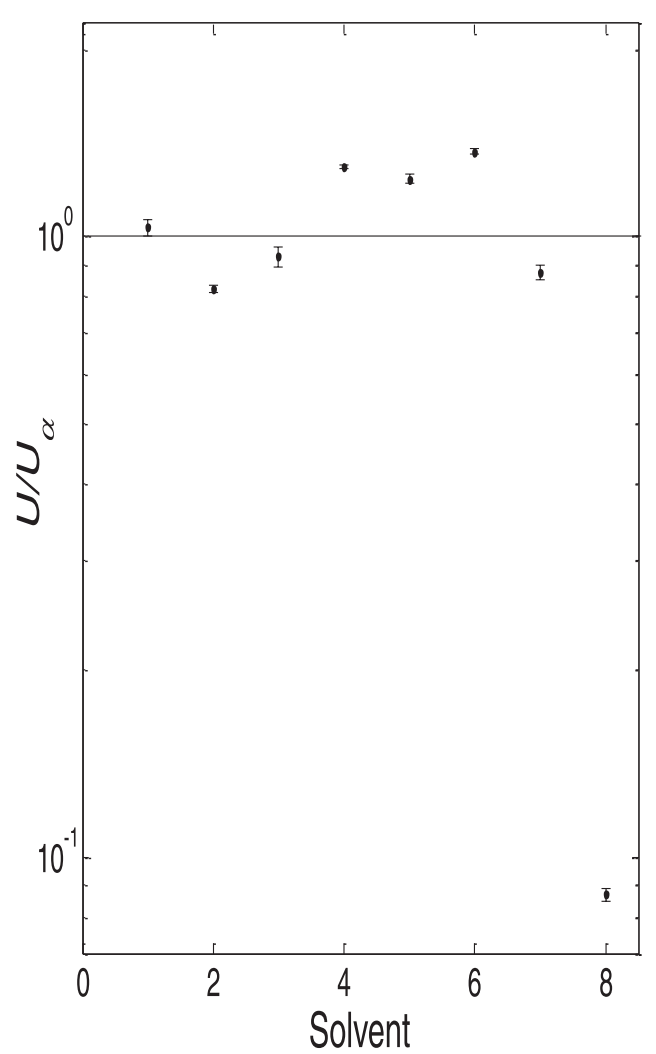

FIG. 4. A comparison of the measured $U$ to the Cohen-Turnbull energy $U_{\alpha}$ for each of the solvents of Table I. Water is in the lower right corner.

sic minimum at $4{ }^{\circ} \mathrm{C}$ and increases with falling temperature throughout most of the supercooled phase, so that $\alpha<0$ therein. Thus for water it does not seem appropriate to use the very small van der Waals radius in calculating the free volume. One concludes that water has a "zero-point" free volume due to hydrogen bonding that is far greater than the van der Waals volume, even at temperatures approaching the glass transition. With water it appears more appropriate to use the room-temperature specific volume $\frac{M}{\rho N_{A}}$ as the mean volume per molecule, rather than an average of the room-temperature and van der Waals volumes. If we employ this in calculating $U_{\alpha}$, the ratio $U / U_{\alpha}$ doubles, but is still a factor of 6 less than unity.

In calculating the average free volume, Cohen and Turnbull ${ }^{4}$ treat the solvent as a system of hard spheres, with no energy penalty if the molecular free volumes are redistributed. In water at a particular instant the MR molecule sees a relatively rigid molecular structure anchored by the hydrogen bonding of each water molecule to 3-4 of its neighbors, so the flowing hard sphere approach might not be appropriate. With water it seems possible that, instead of waiting for the creation of a rare void within the hydrogen-bonded structure large enough for the entire MR molecule to fit within, MR will move into a slot between water molecules equal to or larger than its effective planar thickness. The planar thickness is measured perpendicular to the page in Fig. 1, allowing for the fact that not all the tetrahedral bonds are in the same plane. The slot width expands with increasing temperature as usual, so that Eq. (4) is retained. We believe that the effective planar thickness could be reduced by a factor of order $6^{1 / 3}$ 
from the mean MR radius of Fig. 1. This would then reduce $\gamma$ $v^{*}$ and hence $U_{\alpha}$ by a factor of 6 , so that $U_{\alpha}$ would agree with our measured $U$.

As a first test of the zero-point free volume idea, we measured the diffusion in water of methyl orange instead of methyl red. Methyl orange is somewhat longer than ortho methyl red due to the sulfur trioxide present at the para position. The molecular weights of methyl orange and methyl red are 327 and $269 \mathrm{~g} / \mathrm{mole}$, respectively. We found, however, that $U$ for methyl orange was $5 \%$ less than that of methyl red. This seems consistent with the zero-point free volume approach, since methyl orange and methyl red will have similar planar thicknesses.

Using proton spin echo, Krynicki ${ }^{33}$ et al. found that selfdiffusion in water could also be described by the CohenTurnbull Eq. (6), with a $U$ that is about $32 \%$ of our experimental value for MR diffusion. Price et $a .^{34}$ also found that self-diffusion in water could be reasonably well described by Cohen-Turnbull above $242 \mathrm{~K}$. It is reasonable that the Krynicki et al. experimental $U$ is smaller than ours because of the smaller size of water compared to both the mean radius and the planar thickness of MR, so that $\gamma v^{*}$ is smaller in water. Cohen and Turnbull ${ }^{4}$ state explicitly that with freevolume diffusion, tracer diffusion will have a larger $U$ than self diffusion whenever the tracer is larger than the solvent.

We have proposed that an MR plane will diffuse though a slot in contiguous water molecules, or equivalently that the slot moves over the plane. This presumably requires that the water molecules and MR have fairly specific relative orientations. If so, it might be possible to use proton spin echo in samples of water doped with MR to test the possibility of this "slot diffusion." Relative motion between MR and water should occur with the MR magnetic field not changing a great deal temporally as seen by the water. The spin-echo results can be compared to non-planar solutes, with magnetic fields that rotate as the water molecules translate. We are not aware of any measurements of this type in the current literature.

\section{CONCLUSIONS}

We have found that in all nine solvents investigated (Table I plus toluene), the temperature dependence of $D$ follows the Cohen-Turnbull prediction of Eq. (6) mathematically, and in seven of the solvents the measured $U$ and the Cohen-Turnbull energy are in reasonable agreement. This lends credence to the predictive power of the Cohen-Turnbull theory. We find that tracer diffusion in water also follows Eq. (6), but with a measured energy $U$ that is more than an order of magnitude smaller than the Cohen-Turnbull energy. The most important practical consequence is that tracer diffusion in water will change far less drastically with temperature compared to pure free-volume diffusion. For a temperature change from $20^{\circ} \mathrm{C}$ to $30^{\circ} \mathrm{C}$, the observed diffusion coefficient of MR in water increases by about $30 \%$. If water instead obeyed pure free-volume diffusion, the increase would be a factor of 40 . We have proposed that the relatively rigid "zeropoint" free volume structure forced onto water by hydrogen bonding accounts for the large difference. With water it is important to understand how the mathematical agreement with free-volume theory that we and Krynicki et al. have observed is consistent with the hydrogen bonding. We will be able to continue work in our laboratory on this project after remodeling in the laboratory building has been completed in late 2104. The most important additional experiments will be (1) to test the zero-point idea by using a more spherical solute of about the same volume as MR, which should have a larger $U$ and (2) to measure the diffusion of MR though a solvent with a small molecular size and/or diminutive thermal expansion, but weaker hydrogen bonding than water.

\section{ACKNOWLEDGMENTS}

We are grateful to Dr. Nancy Mills and Dr. Taihyun Chang for helpful conversations. This work was supported under NSF Research at Undergraduate Institutions number 1205313.

${ }^{1}$ P. A. Egelstaff, An Introduction to the Liquid State (Academic Press, London, 1967).

${ }^{2}$ R. B. Bird, W. E. Stewart, and E. N. Lightfoot, Transport Phenomena, 2nd ed. (J. Wiley and Sons, New York, 2007).

${ }^{3}$ H. J. V. Tyrrell and K. R. Harris, Diffusion in Liquids (Butterworths, London, 1984).

${ }^{4}$ M. H. Cohen and D. Turnbull, J. Chem. Phys. 31, 1164 (1959).

${ }^{5}$ D. Turnbull and M. H. Cohen, J. Chem. Phys. 34, 120 (1961).

${ }^{6}$ D. Turnbull and M. H. Cohen, J. Chem. Phys. 52, 3038 (1970).

${ }^{7}$ G. S. Grest and M. H. Cohen, Adv. Chem. Phys. 48, 455 (1981).

${ }^{8}$ F. Reif, Fundamentals of Statistical and Thermal Physics (Waveland Press, Long Grove, IL, 1965).

${ }^{9}$ P. F. F. Almeida, W. L. C. Vaz, and T. E. Thompson, Biochemistry 31, 6739 (1992).

${ }^{10}$ T.-X. Xiang, J. Chem. Phys. 109, 7876 (1998).

${ }^{11}$ F. Faupel, W. Frank, M.-P. Macht, H. Mehrer, V. Naundorf, K. Rätzke, H. R. Schober, S. K. Sharma, and H. Teichler, Rev. Mod. Phys. 75, 237 (2003).

${ }^{12}$ A. E. Nasrabad, J. Chem. Phys. 130, 024503 (2009); A. E. Nasrabad, R. Lagaei, and B. C. Eu, J. Phys. Chem. B 109, 8171 (2005).

${ }^{13}$ M. Petrowsky and R. Frech, J. Phys. Chem. B 114, 8600 (2010).

${ }^{14}$ D. W. Pohl, S. E. Schwarz, and V. Irniger, Phys. Rev. Lett. 31, 32 (1973).

${ }^{15}$ H. Eichler, G. Salje, and H. Stahl, J. Appl. Phys. 44, 5383 (1973).

${ }^{16}$ H. J. Eichler, P. Günter, and D. W. Pohl, Laser Induced Dynamic Gratings (Springer-Verlag, Berlin, 1986).

${ }^{17}$ D. R. Spiegel, M. B. Sprinkle, and T. Chang, J. Chem. Phys. 104, 4920 (1996).

${ }^{18}$ D. R. Spiegel, A. H. Marshall, N. T. Jukam, H. S. Park, and T. Chang, J. Chem. Phys. 109, 267 (1998).

${ }^{19}$ M. Terazima, K. Okamoto, and N. Hirota, J. Phys. Chem. 97, 5188 (1993).

${ }^{20}$ H. S. Park, K. S. Oh, K. S. Kim, T. Chang, and D. R. Spiegel, J. Phys. Chem. B 103, 2355 (1999).

${ }^{21}$ T. Chang, H. Kim, and H. Yu, Chem. Phys. Lett. 111, 64 (1984).

${ }^{22}$ W. Köhler, J. Chem. Phys. 98, 660 (1993).

${ }^{23}$ F. Mallamace, C. Cosaro, and H. E. Stanley, Proc. Natl. Acad. Sci. U.S.A. 110, 4899 (2013).

${ }^{24}$ P. B. Macedo and T. A. Litovitz, J. Chem. Phys. 42, 245 (1965).

${ }^{25}$ V. Holten and M. A. Anisimov, Sci. Rep. 2, 713 (2012).

${ }^{26}$ H. Tanaka, Eur. Phys. J. E 35, 113 (2012).

${ }^{27}$ J. D. Smith, C. D. Cappa, K. R. Wilson, R. C. Cohen, P. L. Geissler, and R. J. Saykally, Proc. Natl. Acad. Sci. U.S.A. 102, 14171 (2005).

${ }^{28}$ P. Debenedetti and H. E. Stanley, Phys. Today 56(6), 40 (2003).

${ }^{29}$ K. T. Gillen, D. C. Douglass, and M. J. R. Hoch, J. Chem. Phys. 57, 5117 (1972).

${ }^{30}$ C. A. Angell, E. D. Finch, L. A. Woolf, and P. Bach, J. Chem. Phys. 65, 3063 (1976).

${ }^{31}$ F. X. Prielmeier, E. W. Lang, R. J. Speedy, and H.-D. Lüdemann, Phys. Rev. Lett. 59, 1128 (1987).

${ }^{32}$ T. V. Lokotosh, S. Magazu, G. Maisano, and N. P. Malomuzh, Phys. Rev. E 62, 3572 (2000).

${ }^{33}$ K. Krynicki, C. D. Green, and D. W. Sawyer, Faraday Discuss. Chem. Soc. 66, 199 (1978).

${ }^{34}$ W. S. Price, H. Ide, and Y. Arata, J. Phys. Chem. A 103, 448 (1999). 